\title{
Neutrino decay and Solar Neutrino Seasonal effect
}

\section{R. Picoreti}

Instituto de Física Gleb Wataghin - UNICAMP, 13083-859, Campinas SP, Brazil

\section{M. Guzzo}

Instituto de Física Gleb Wataghin - UNICAMP, 13083-859, Campinas SP, Brazil

\section{P. C. de Holanda}

Instituto de Física Gleb Wataghin - UNICAMP, 13083-859, Campinas SP, Brazil

\section{Orlando Peres ${ }^{* \dagger}$}

Instituto de Física Gleb Wataghin - UNICAMP, 13083-859, Campinas SP, Brazil

The Abdus Salam International Centre for Theoretical Physics, Strada Costiera 11, 34014

Trieste, Italy

E-mail: orlandodifi.unicamp.br

\begin{abstract}
We consider the possibility of solar neutrino decay as a sub-leading effect on their propagation between production and detection. Using current oscillation data, we set a new lower bound to the $v_{2}$ neutrino lifetime at $\tau_{2} / m_{2} \geq 7.2 \times 10^{-4} \mathrm{~s} . \mathrm{eV}^{-1}$ at $99 \%$ C.L.. Also, we show how seasonal variations in the solar neutrino data can give interesting additional information about neutrino lifetime.
\end{abstract}

38th International Conference on High Energy Physics

3-10 August 2016

Chicago, USA

\footnotetext{
* Speaker.

${ }^{\dagger}$ Funding FAPESP funding grant 2012/16389-1, 2014/19164-6 and 2016/08308-2.
} 


\section{Introduction}

The question if the neutrino decay is as old as the idea of neutrino oscillations. Now after oscillation was confirmed in different experiments [1] it is interesting time to look again for the possibility that the neutrino decay.

The decay scenario is possible when we introduce a imaginary term in the Hamiltonian, $\mathrm{E} \rightarrow \mathrm{E}$ $-i \Gamma / 2$ for neutrino mass eigenstate. The parameter $\Gamma$ in the lab system is given by $\Gamma_{\text {lab }}=\equiv \frac{m}{\tau E}$ where $\mathrm{E}$ is the neutrino energy. For the solar neutrino oscillations the more relevant mass eigenstates are the states 1 or 2 . The state 3 is less relevant due the smallness of $\theta_{13}$ mixing parameter compared with other mixing parameters. Then we will focus on the decay parameter of mass eigenstate 2 . For now on we will use $\frac{\tau_{2}}{m_{2}}$ as the decay parameter.

Some previous constrains on $\frac{\tau}{m}$ came from previous analysis on solar neutrino data only. For solar neutrinos, the decay of the mass-eigenstate $v_{2}$ into the lighter state $v_{1}$ is disfavored by the data and the current bound to $v_{2}$ lifetime for invisible non-radiative decays [2] is $\tau_{2} / m_{2} \geq 8.7 \times$

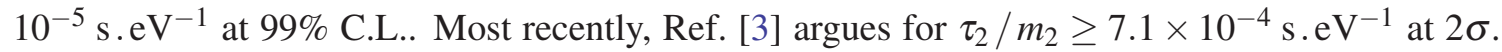
Constrains for the other decay parameter, $\tau_{3} / m_{3}$ are possible $[4,5]$.

\section{Formalism}

After production in the solar core, neutrinos propagate outwards undergoing flavor oscillation and resonant flavor transition due to the solar matter potential. After emerging from the Sun, they travel across the interplanetary medium until they reach the Earth's surface where they can be detected either promptly or after traversing Earth's matter — on which they may also be subject to matter effects.

The transition amplitude for an electron neutrino produced in the Sun to be detected on Earth as a neutrino of flavor $\alpha, v_{e} \rightarrow v_{\alpha}$, for the standard case of neutrino oscillations with MSW effect, can be written as [6]

$$
A_{e \alpha}=\sum A_{e i}^{\odot} A_{i i}^{\mathrm{vac}} A_{i \alpha}^{\oplus},
$$

where $A_{e i}^{\odot}$ is the transition amplitude of an electron neutrino produced in the solar core to be in a $v_{i}$ mass-eigenstate in the solar surface, $A_{i i}^{\mathrm{vac}}$ is the transition amplitude for the propagation between Sun and Earth surfaces, and $A_{i \alpha}^{\oplus}$ is the transition amplitude of a $v_{i}$ to be in a $v_{\alpha}$ state upon detection on Earth.

The transition probability is given as $P\left(v_{e} \rightarrow v_{\alpha}\right)=\left|A_{e \alpha}\right|^{2}$. In the LMA parameter region one can neglect coherence effects [7] and simply write the incoherent sum of probabilities:

$$
P\left(v_{e} \rightarrow v_{\alpha}\right)=\sum_{i} P_{e i}^{\odot} P_{i m / t a u}^{\oplus},
$$

where $P_{e i}^{\odot}=\left|A_{e i}^{\odot}\right|^{2}$ is the probability of the produced $v_{e}$ to be found as a $v_{i}$ at the surface of the Sun, and $P_{i \alpha}^{\oplus}=\left|A_{i \alpha}^{\oplus}\right|^{2}$ is the probability of a $v_{i}$ to be detected as a $v_{\alpha}$ on Earth.

Considering the current limits to their lifetime, neutrinos do not decay inside the Sun and it is sufficient to consider their decay on their way to Earth. The survival probability for the invisible decay of a neutrino mass-eigenstate $i$, with energy $E_{v}$, after propagating a distance $L$, is

$$
P_{\mathrm{i}}^{\text {surv }}=\exp \left[-\left(\frac{m_{i}}{\tau_{i} E_{v}}\right) L\right]
$$


where $m_{i}$ is the eigenstate mass, $\tau_{i}$ is the eigenstate lifetime and $L$ is the Sun-Earth distance.

For the assumption that only the $v_{2}$ mass-eigenstate is unstable, the electron neutrino survival probability including decay and oscillation for three neutrino families is

$$
P\left(v_{e} \rightarrow v_{e}\right)=c_{13}^{4}\left[P_{e 1}^{\odot} P_{1 e}^{\oplus}+P_{e 2}^{\odot}\left(P_{2}^{\text {surv }}\right) P_{2 e}^{\oplus}\right]+s_{13}^{4},
$$

where $s_{i j}=\sin \theta_{i j}$ and $c_{i j}=\cos \theta_{i j}$ and $P_{\mathrm{i}}^{\text {surv }}$ is given in Eq. (2.3) and $P_{e i}^{\odot}$ and $P_{i e}^{\oplus}$ are the probabilities in Eq. (2.2).

\section{Seasonal Effect}

One interesting consequence of the decay scenario that has was not discussed before is the increase of the seasonal variation of solar neutrino flux. In the absence of decay, the neutrino flux arriving on Earth is given by $\phi_{v}^{\oplus}=\phi_{v}^{\odot} /\left(4 \pi r^{2}\right)$, where $r=r(t)$ is the time-dependent EarthSun distance. The ratio between maximum (at perihelion) and minimum (at aphelion) fluxes is $R_{0}=\left(1+\varepsilon_{0}\right)^{2} /\left(1-\varepsilon_{0}\right)^{2}$, where $\varepsilon_{0}=0.0167$ is the eccentricity of Earth's orbit. Due the $1 / r^{2}$ factor the neutrino flux have a seasonal dependence and different solar neutrino experiments have searched for the seasonal effect as you can see in the left side of Figure (1).

When the neutrino decay the neutrino survival probability, Eq. (2.4) have an additional dependence on the distance due the $P_{2}^{\text {surv }}$ factor. In the aphelion (perilhelion) the distance earth-sun is the smallest (largest) distance. Then this implies that $P_{2}^{\text {surv }}$ factor is respectivelly bigger (smaller) compared with the value for the average distance. This implies that we increase the eccentricity in the decay scenario compared with the no decay scenario. In the decay scenario the ratio between the rates at aphelium and (perihelion is

$$
R=R_{0} \frac{N\left(r_{\mathrm{per}}\right)}{N\left(r_{\mathrm{aph}}\right)}=\frac{(1+\varepsilon)^{2}}{(1-\varepsilon)^{2}}
$$

where $r_{\text {aph }}\left(r_{\text {per }}\right)$ is the aphelion (perihelion) distance and $N$ is the number of events calculated from the convolution of the adequate probabilities and cross sections for each experiment. This implies that $R>R_{0}$ for all energies and thus, for any neutrino decay scenario, an enhancement in the seasonal variation of the solar neutrino flux would be expected.

Thus, the measurement of an eccentricity $\varepsilon>\varepsilon_{0}$ is a hint in the direction of the neutrino decay scenario.

\section{Results}

Using the electron survival probability we can compute the rates and spectral information for the solar neutrino data. Details of data used are listed in Ref. ([12]). In the decay scenario, beside

Figure 1: Left: Experimental values for $\varepsilon / \varepsilon_{0}$. Black lines are the best-fit values and darker (lighter) shades are the $1 \sigma(2 \sigma)$ ranges. Right: Dependence of the orbital eccentricity $\varepsilon$ with the neutrino lifetime $\tau_{2} / m_{2}$ as it would be measured by different experiments — the ${ }^{7} \mathrm{Be}$ line in Borexino (BOR), and the ${ }^{8} \mathrm{~B}$ spectrum in Super-Kamiokande (SK) and SNO. 
the usual oscillation parameters $\theta_{12}, \Delta m_{12}^{2}$ and $\theta_{13}$ we have the decay parameter $\alpha$. If we stay only on solar neutrino data there is a degenerescence between $\theta_{! 3}$ and $\Delta m_{12}^{2}$ that can be lifted using the information from Baya Bay experiment. Beside that the KamLand experiment provides constrains on $\Delta m_{12}^{2}$ that are much more constrained then from solar neutrino data. The solution for these degenerescences and more precise experiments is to include theirs data from KamLand and Daya Bay as well into the analysis.

From past analysis we know already that the constrains on the decay parameter $\tau / m$ already implies that experiments with distances smaller then the sun-earth distance are not affected by the decay scenario. Then the experimental analysis of Daya Bay and KamLand experiments can were computed for the stable neutrino scenario can be equally used for the decay scenario. The result is

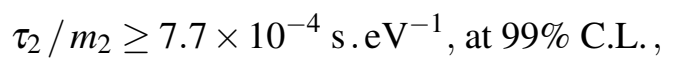

For the seasonal effect we compute the change in the effective eccentricity parameter due decay for different experiments. The results can be seen in the right side of Figure 1. We include this constrains on our analysis. The combined solar neutrino data, KamLand, Daya Bay and seasonal data give the following weaker constrain

$$
\tau_{2} / m_{2} \geq 7.2 \times 10^{-4}{\mathrm{~s} . \mathrm{eV}^{-1}} \text {, at } 99 \% \text { C.L. }
$$

\section{Conclusion}

We revisit the hypothesis of neutrino decay for solar neutrinos and discover that this scenario produce a distinct seasonal effect on solar neutrinos. From solar neutrino data combined with KamLand and Daya Bay data we get the decay parameter to be $\tau_{2} / m_{2} \geq 7.2 \times 10^{-4}{\mathrm{~s} . V^{-1}}^{-1}$ at $99 \%$ C.L. Putting these constrain on the $P_{2}^{\text {surv }}=\exp \left[-\left(\frac{m_{2}}{\tau_{2} E_{v}}\right) L\right]<\exp \left[9 . \times 10^{-2}\right]$ for a $10 \mathrm{MeV}$ neutrino energy. This implies that for all range of solar neutrino data the constrain obtain in this paper out the decay as a sub-leading effect.

The use of the results of different experiments allows us for the first time to broken the degenerescence of neutrino oscillation parameters and to achieve the best known limit for neutrino instability for the lighter mass eigenstate [13].

\section{References}

[1] Nobel Prize for T. Kajita and A. K. McDonald

[2] A. Bandyopadhyay, S. Choubey, S. Goswami, Phys.Lett. B555 (2003) 33-42.

[3] J. M. Berryman, A. de Gouvea and D. Hernandez, Phys. Rev. D 92, no. 7, 073003 (2015) doi:10.1103/PhysRevD.92.073003 [arXiv:1411.0308 [hep-ph]].

[4] T. Abrahao, H. Minakata, H. Nunokawa and A. A. Quiroga, JHEP 1511, 001 (2015) doi:10.1007/JHEP11(2015)001 [arXiv:1506.02314 [hep-ph]].

[5] R. A. Gomes, A. L. G. Gomes and O. L. G. Peres, Phys. Lett. B 740, 345 (2015) doi:10.1016/j.physletb.2014.12.014 [arXiv:1407.5640 [hep-ph]].

[6] A. S. Joshipura, E. Masso, S. Mohanty, Phys.Rev. D66 (2002) 113008. 
[7] A. S. Dighe, Q. Y. Liu and A. Y. Smirnov, hep-ph/9903329.

[8] B. T. Cleveland, Astrophys.J. 496 (1998) 505-526.

[9] W. Hampel, et al., Phys.Lett. B447 (1999) 127-133.

[10] J. N. Abdurashitov, et al., Phys.Rev. C80 (2009) 015807.

[11] M. Altmann, et al., Phys.Lett. B616 (2005) 174-190.

[12] P. C. de Holanda, A. Yu. Smirnov, Phys.Rev. D83 (2011) 113011.

[13] R. Picoreti, M. M. Guzzo, P. C. de Holanda and O. L. G. Peres, Phys. Lett. B 761, 70 (2016) doi:10.1016/j.physletb.2016.08.007 [arXiv:1506.08158 [hep-ph]]. 